\title{
Vibration Model Creation and Solution of Bending-Torsion-Axial-Swing Coupled Helical Gear Transmission System Based on Lumped Parameter Approximation Method
}

\author{
Li Chang ${ }^{1, a}$,Han Xing ${ }^{1, b}$ \\ ${ }^{1}$ School of Mechanical Engineering \& Automation, University of Science and Technology Liaoning, \\ Anshan 114051, China. \\ alichang2323-23@163.com, ${ }^{b}$ hanxinggctx@163.com
}

Keywords: Helical gear; Lumped parameter approximation method; Vibration response

Abstract. Based on comprehensive considerations of influences generated by stiffness excitation, deviation excitation, meshing impact excitation, friction of tooth surface, and other kinds of nonlinear factors, it established a nonlinear coupled vibration model of bending-torsion-axial-swing coupled helical gear transmission system by applying the Lumped Mass Method. After transformed the model to dimensionless form, it used Runge-Kutta method to solve the nonlinear vibration model of the system, and then the time domain chart, spectrum chart, phase chart, Poincare chart, and FFT chart were obtained; it discussed the influence of system parameters on its dynamic characteristics.

\section{Introduction}

Gear transmission systems had been widely used in machinery, metallurgy, mining, automobile, spaceflight and other fields. With the development of modern industries and mechanical science and technologies, requirements for gear transmission system with quick running, light duty, large load-bearing capacity, long service, and high reliability had been desired, this made it inevitable for system to work under the conditions of high speed, heavy duty, high temperature, and approaching to limit load for a long time, so it was a rigorous requirement of gear transmission accuracy, vibration , and noise characteristics[1].

The creation of vibration model of bending-torsion-axial-swing coupled helical gear transmission system

Creation of Vibration Model. Because of elastic deformation of transmission shaft and its support, the helical gear transmission system did not only produce bending vibration, torsion vibration, axial vibration but also produce torsion pendulum vibration, in order to take a further research of dynamic characteristics of the transmission system, it needed to establish a nonlinear coupling vibration model for bending-torsion-axial-swing coupled of helical gear system, showed as Fig.1.

Every gear had five degrees of freedom; $c_{m}$ and $k_{h}$ were damping and time varying contact rigidity; $m_{i j}$ and $c m_{i j}(j=x, y, z)$ were supporting rigidity and equivalent damping; $e(t)$ was static error. $\beta$ was driving gear helix angle, $\alpha$ was transverse pressure angle, the $x, y$, and $z$ direction components were[2]:

$\left\{\begin{array}{lll}k_{m x}=k_{h} \sin \alpha & k_{m y}=k_{h} \cos \alpha \cos \beta & k_{m z}=k_{h} \cos \alpha \sin \beta \\ c_{m x}=c_{m} \sin \alpha & c_{m y}=c_{m} \cos \alpha \cos \beta & c_{m z}=c_{m} \cos \alpha \sin \beta \\ e_{x}=e \sin \alpha & e_{y}=e \cos \alpha \cos \beta & e_{z}=e \cos \alpha \sin \beta\end{array}\right.$

Radial dynamic meshing force $F_{x}$ was:

$$
\begin{aligned}
& F_{x}=k_{m x} \sin \alpha \cdot f\left[x_{p}-x_{g}-\left(y_{p}+\theta_{p z} R_{p}+y_{g}-\theta_{g z} R_{g}\right) \tan \alpha_{t}-e_{x}\right]+c_{m} \sin \alpha\left[\frac{\alpha}{p}-\alpha_{g}-\right. \\
& \left.\left(\oint_{p}+\theta_{p z}^{\alpha} R_{p}+\oint_{g}-\theta_{g z}^{\alpha} R_{g}\right) \tan \alpha_{t}-\phi_{x}\right]
\end{aligned}
$$

Tangential dynamic meshing force $F_{y}$ was:

$$
F_{y}=k_{m y} \cos \alpha \cos \beta \cdot f\left[y_{p}-y_{g}+\theta_{p z} R_{p}+\theta_{g z} R_{g}-e_{y}\right]+c_{m} \cos \alpha \cos \beta\left[\phi_{p}-\hat{\gamma}_{g}+\theta_{p z}^{\alpha} R_{p}+\theta_{g z}^{\alpha} R_{g}-\oint_{y}\right]
$$


Axial dynamic meshing force $F_{z}$ was:

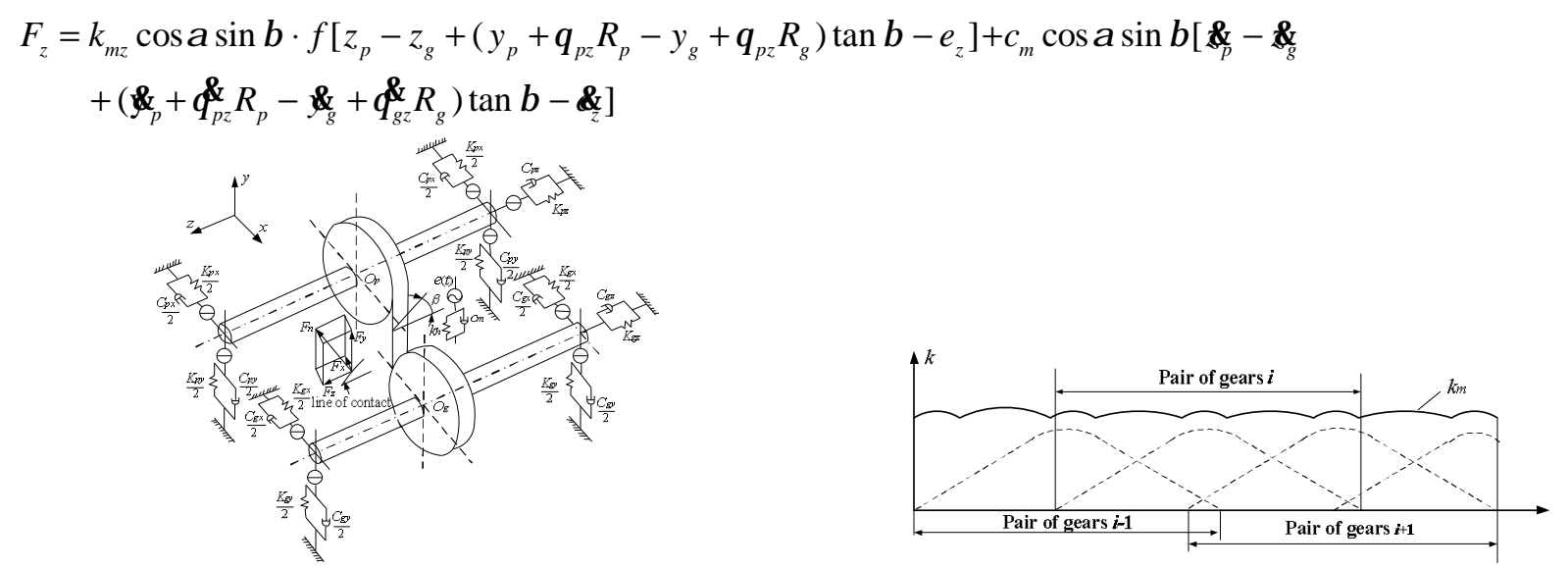

Fig.1 The helical gear dynamics analysis model Fig.2 The time-varying meshing stiffness of helical gears

With the method of Newton second law the helix gear pair calculation equations were:

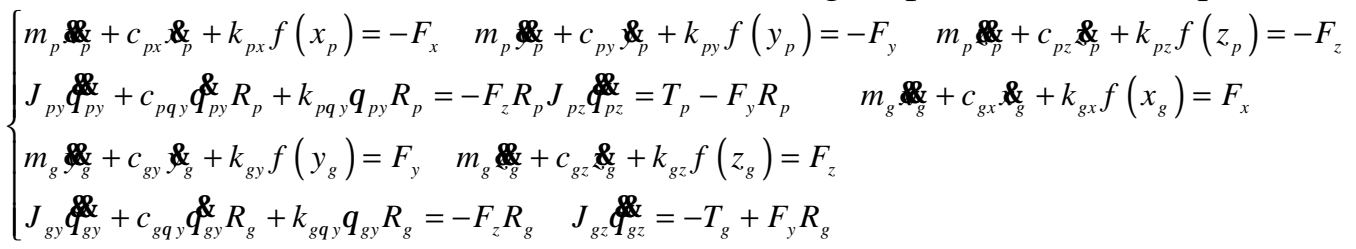

Where, $R_{i}(i=p, g)$ was gear base radius; $m_{i}(i=p, g)$ and $J_{i j}(i=p, g ; j=y, z)$ were gears masses and rotary inertias; $c_{i j}(i=p, g ; j=x, y, z)$ and $k_{i j}(i=p, g ; j=x, y, z)$ were gears supporting damping and rigidity separately; $c_{i \theta y}(i=p, g)$ and $k_{i \theta y}(i=p, g)$ were torsion damping and rigidity; $f\left(i_{j}\right)(i=x, y, z ; j=p, g, s)$ was backlash non-linear function; $b_{5}$ was half of backlash, $b_{1}$ and $b_{2}$ were half of gears radial internal clearances, and $2 b_{3}$ and $2 b_{4}$ were half of gears axial internal clearances.

Comparing with spur gear meshing, helix gear mesh curve did not appear step mutation, while it performed a character of "point-line-point" periodic variation and was showed as Fig.2[3].

Where, $k_{m}$ was mean mesh rigidity; $a_{n}$ and $b_{n}$ were Fourier expansion coefficients, and $n=1,2, \cdots, N$.

Dimensionless dealing of the model. Given gear rigidity coefficient order varied from $10^{7}$ to $10^{8}$ and damping ratio order varied from $10^{-4}$ to $10^{-1}$, while vibration response changed from $10^{0} \mu \mathrm{m}$ to $10^{1}$ $\mu \mathrm{m}$, so it needed to make the Equ.2 indexes being dimensionless. The helical gear pair natural frequency $\omega_{n}=\sqrt{k_{m} / m_{e}}$ ( $m_{e}$ was equivalent mass), given displacement scale $b_{5}$ and dimensionless time $\tau=t \cdot \omega_{n}, \quad$ and 2 order and 3 order derived numbers against $\tau$. The dimensionless equation system was

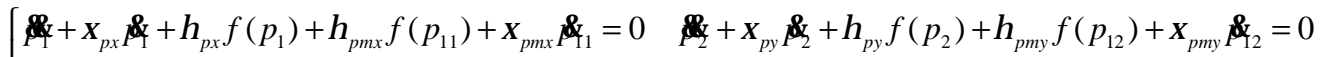

$$
\begin{aligned}
& +\xi_{p z}+\eta_{p z} f\left(p_{3}\right)+\eta_{p m z} f\left(p_{13}\right)+\xi_{p m z}=0 \quad+\xi_{p \theta y}+\eta_{p \theta y} p_{4}+4 \eta_{p m z} f\left(p_{13}\right)+4 \xi_{p m z}=0
\end{aligned}
$$

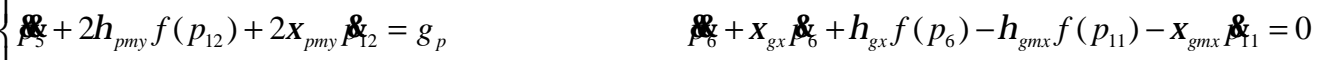

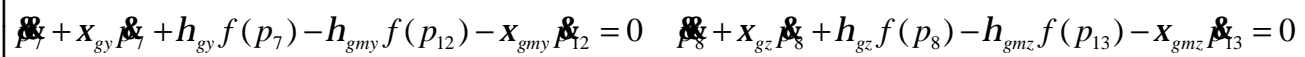

$$
\begin{aligned}
& +\xi_{g \theta y}+\eta_{g \theta y} p_{9}+4 \eta_{g m z} f\left(p_{13}\right)+4 \xi_{g m z}=0 \quad-2 \eta_{g m y} f\left(p_{12}\right)-2 \xi_{g m y} \int_{2}=-g_{g}
\end{aligned}
$$

Where, dimensionless damping indexes were $\xi_{p x}=\frac{c_{p x}}{m_{p} \omega_{n}}, \xi_{p y}=\frac{c_{p y}}{m_{p} \omega_{n}} \quad, \quad \xi_{p z}=\frac{c_{p z}}{m_{p} \omega_{n}}$, $\xi_{p \theta y}=\frac{2 c_{p x}\left(l_{1}+l_{2}\right)}{m_{p} R_{p} \omega_{n}}, \xi_{p m x}=\frac{c_{m} \sin \alpha}{m_{p} \omega_{n}}, \xi_{p m y}=\frac{c_{m} \cos \alpha \cos \beta}{m_{p} \omega_{n}}, \xi_{p m z}=\frac{c_{m} \cos \alpha \sin \beta}{m_{p} \omega_{n}}, \xi_{g x}=\frac{c_{g x}}{m_{g} \omega_{n}}, \xi_{g y}=\frac{c_{g y}}{m_{g} \omega_{n}}$, $\xi_{g z}=\frac{c_{g z}}{m_{g} \omega_{n}}, \xi_{g \theta y}=\frac{2 c_{g x}\left(l_{3}+l_{4}\right)}{m_{g} R_{g} \omega_{n}}, \xi_{g m x}=\frac{c_{m} \sin \alpha}{m_{g} \omega_{n}}, \xi_{g m y}=\frac{c_{m} \cos \alpha \cos \beta}{m_{g} \omega_{n}}, \xi_{g m z}=\frac{c_{m} \cos \alpha \sin \beta}{m_{g} \omega_{n}} \circ$ Dimensionless rigidity indexes were $\eta_{p x}=\frac{\omega_{p x}^{2}}{\omega_{n}^{2}}, \eta_{p y}=\frac{\omega_{p y}^{2}}{\omega_{n}^{2}}, \eta_{p z}=\frac{\omega_{p z}^{2}}{\omega_{n}^{2}}, \eta_{p \theta y}=\frac{2 \omega_{p x}^{2}\left(l_{1}+l_{2}\right)}{R_{p} \omega_{n}^{2}}, \eta_{p m x}=[1+s \cdot \cos (\omega \tau)] \frac{m_{e} \sin \alpha}{m_{p}}, \eta_{g x}=\frac{\omega_{g x}^{2}}{\omega_{n}^{2}}, \eta_{g y}=\frac{\omega_{g y}^{2}}{\omega_{n}^{2}}$, 
$\eta_{p m y}=[1+s \cdot \cos (\omega \tau)] \frac{m_{e} \cos \alpha \cos \beta}{m_{p}}, \eta_{g z}=\frac{\omega_{g z}^{2}}{\omega_{n}^{2}}, \eta_{p m z}=[1+s \cdot \cos (\omega \tau)] \frac{m_{e} \cos \alpha \sin \beta}{m_{p}}, \eta_{g \theta y}=\frac{2 \omega_{g x}^{2}\left(l_{3}+l_{4}\right)}{R_{g} \omega_{n}^{2}}$,

$\eta_{g m x}=[1+s \cdot \cos (\omega \tau)] \frac{m_{e} \sin \alpha}{m_{g}}, \eta_{g m y}=[1+s \cdot \cos (\omega \tau)] \frac{m_{e} \cos \alpha \cos \beta}{m_{g}}, \eta_{g m z}=[1+s \cdot \cos (\omega \tau)] \frac{m_{e} \cos \alpha \sin \beta}{m_{g}}$

The other dimensionless parameters were[4]:

$\omega_{p x}=\sqrt{k_{p x} / m_{p}}, \omega_{p y}=\sqrt{k_{p y} / m_{p}}, \omega_{p z}=\sqrt{k_{p z} / m_{p}}, \omega_{g x}=\sqrt{k_{g x} / m_{g}}, \omega_{g y}=\sqrt{k_{g y} / m_{g}}, \quad \omega_{g z}=\sqrt{k_{g z} / m_{g}}$,

$g_{p}=\frac{2 T_{p}}{m_{p} R_{p} b_{5} \omega_{n}^{2}}, \quad g_{g}=\frac{2 T_{g}}{m_{g} R_{g} b_{5} \omega_{n}^{2}}, \quad m_{e}=\frac{J_{p} J_{g}}{J_{p} R_{g}^{2}+J_{g} R_{p}^{2}}$

\section{Solving of the gear driven system non-linear dynamic model}

Create a higher-order fitting polynomial for a non-linear function. In a dynamic solution, a clearance non-linear function was expressed as several sectional functions. In a calculation process if sectional function judgment times was too much, this would cause bad computation efficiency even endless loop. Therefore, it expressed the clearance non-linear function as a higher order fitting polynomial.

Fetched uniformly 600 sample points at function curve, and used MATLAB software to program the polynomial basing on least squares theory. Fitting coefficients were listed in Table 1. The higher fitting order, the closer to original function. Once it up to 7 times the curve no longer changed(Fig.3).

Tab. 1 The fitting coefficient of high order curve

\begin{tabular}{cccccc}
\hline Times & $x^{1}$ & $x^{3}$ & $x^{5}$ & $x^{7}$ & $x^{9}$ \\
\hline 1 & 0.5187 & - & - & - & - \\
3 & 0.1725 & 0.0640 & - & - & - \\
5 & -0.0542 & 0.1814 & -0.0117 & - & - \\
7 & -0.1573 & 0.2843 & -0.0368 & 0.0017 & \\
9 & -0.1557 & 0.2818 & -0.0357 & 0.0015 & 0 \\
\hline
\end{tabular}

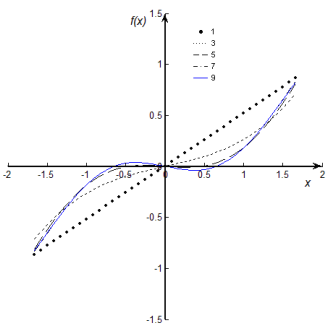

Fig.3 Fitting curves of the nonlinear function

\section{Solving the gear driven system non-linear dynamic model}

Using 5 order variable step self adaptive (Runge-Kutta) method, it could get different system dynamic responses containing displacement-time curve, speed-time curve, phase plane curve, Poincare graph, and FFT graph. Took the gears $x$ and $y$ direction dynamic responses as example, the response curves were showed in Fig.4 and Fig.5.

These results showed that the driving gear vibration amplitude along the $x$ axis (radial) was bigger than the driven gear, the wave frequency was much severer and the vibration smooth was lower than the driven gear; the driving gear vibration was similar to a single periodic simple harmonic vibration, and after a spectrum analysis it showed that low-frequency signals of the driving gear covered the chief ratio; Phase picture was a un-closed curve which had constant breadth, and this showed that these two gears run as non-periodic motions along the $x$ axial; At $y$ (tangential) direction, the driven gear vibration amplitude was bigger than the driving gear, and this mean that the driven gear vibration was much severer; these two gears vibration displacements and speeds were single periodic simple harmonic waves, the Phase pictures were closed curves that had constant widths, and this showed that these two gears run as periodic motions along the $y$ direction. 


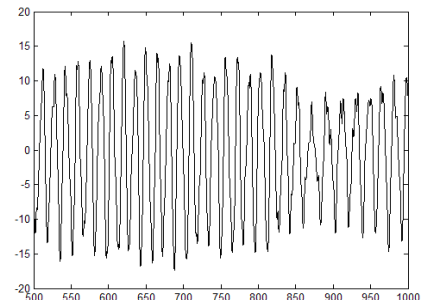

(a)

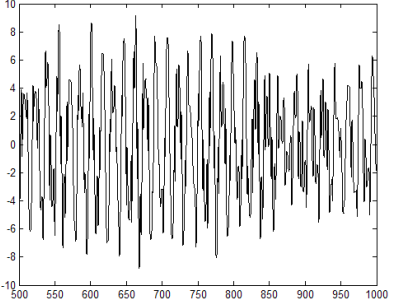

(b)

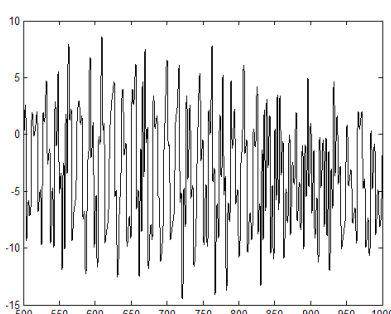

(c)

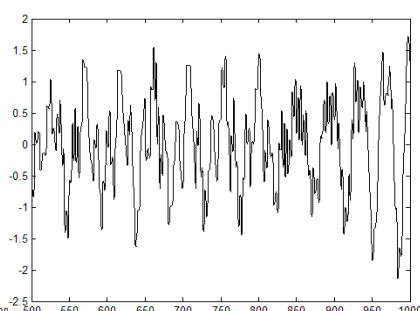

(d)

(a) Driving gear time-displacement response (b) Driven gear time-displacement response

(c)Driving gear time-speed response (d) Driven gear time-displacement response

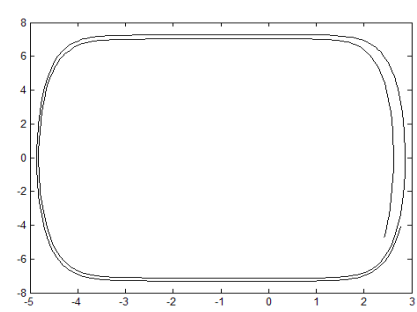

$(e)$

(e) Driving gear phase diagram ( $g$ ) Driving gear FFTdiagram

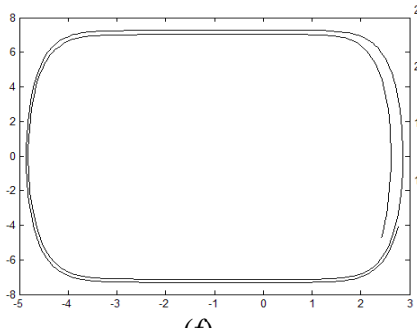

$(f)$

(f) Driven gear phase diagram

(h) Driven gear phase diagram

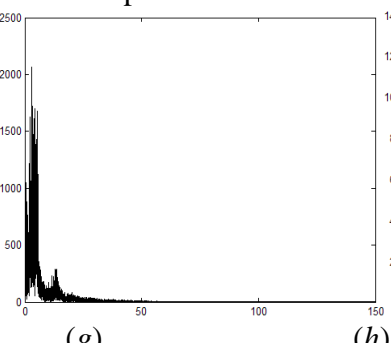

$(g)$

(h)

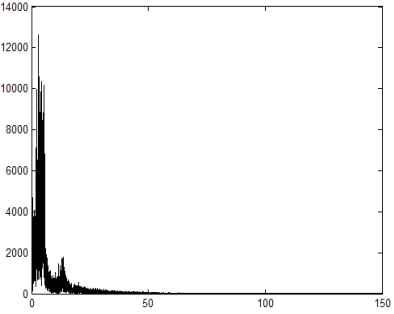

Fig.4(a h) Dynamic responses along x direction of the helix gears

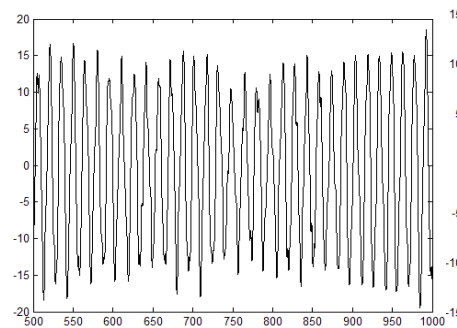

(a)

(a) Driving gear time-displacement response

(c) Driving gear time-speed response

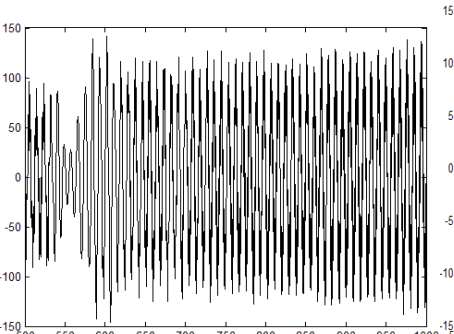

(b)

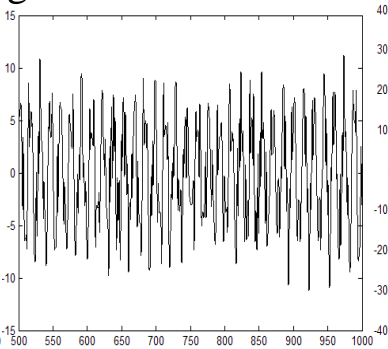

(c)

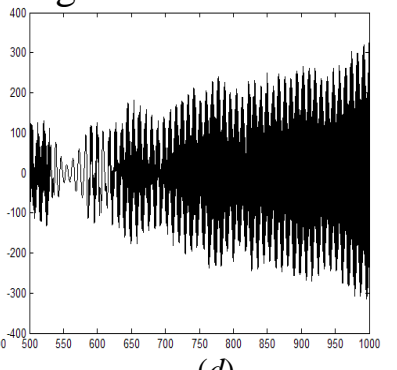

(d)

(b) Driven gear time-displacement response

(d) Driven gear time-displacement response

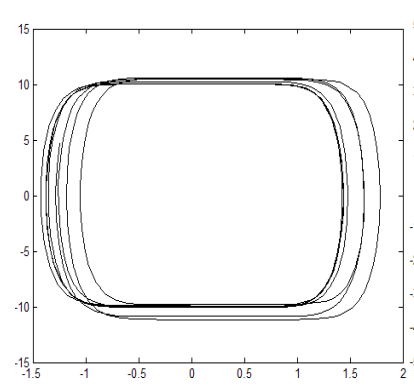

(e)

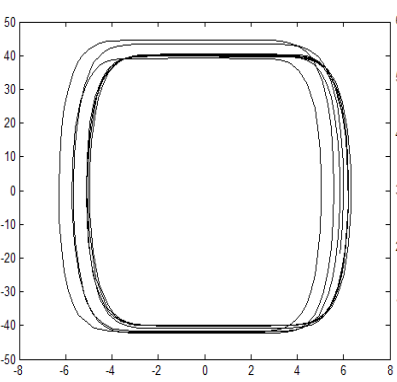

(f)

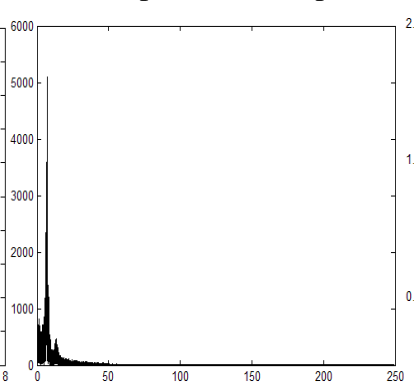

$(g)$

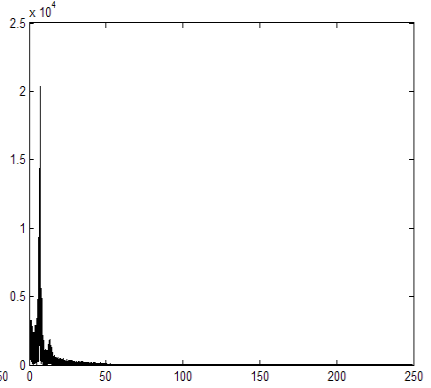

(h)

(e) Driving gear phase diagram

(f) Driven gear phase diagram

(g) Driving gear FFTdiagram

(h) Driven gear phase diagram

Fig.5(a h) Dynamic responses along y direction of the helix gears

\section{Acknowledgements}

This work is financially supported by National Nature Science Foundation of China (E050402/51105187), Program for Liaoning Excellent Talents in University (013202), General program of Liaoning education department(L2014110), and Anshan youth scientific personnel foster program(0000077).

\section{References}

[1] An Weiguang, Cai Yinlin. Reliability analysis and optimistic design of the random mechanism system, Harbin: Publication of Harbin engineering university, 2005:1-2. 
[2] Mechanical reliability design, Shanghai: Publication of Shanghai Jiao Tong University. 1992:12-15.

[3] Li Yongdong. Monte Carlo numerical modeling of a tank gear's contact fatigue strength reliability[J]. Journal of mechanical strength, 2006,28(1):46-50.

[4] Sun Zhili, Chen Liangyu. Reliability design method of practical mechanism, Beijing: Science press, 2003: 215-225. 